\title{
A SEARCH FOR EXTREME-ULTRAVIOLET EMISSION FROM COMETS WITH THE COSMIC HOT INTERSTELLAR PLASMA SPECTROMETER (CHIPS)
}

\author{
T. P. SASSEEN \\ Department of Physics, University of California, Santa Barbara, CA 93106; tims@physics.ucsb.edu \\ M. Hurwitz \\ Space Sciences Laboratory, University of California, Berkeley, CA 94720 \\ C. M. LISSE \\ Applied Physics Laboratory, The Johns Hopkins University, Laurel, MD 20723 \\ V. KHARChEnKo \\ Harvard-Smithsonian Center for Astrophysics, Cambridge, MA 02138 \\ D. Christian \\ Department of Pure and Applied Physics, Queens University, Belfast, UK \\ S. J. WolK \\ Harvard-Smithsonian Center for Astrophysics, Cambridge, MA 02138 \\ M. M. SIRK \\ Space Sciences Laboratory, University of California, Berkeley, CA 94720 \\ AND \\ A. Dalgarno \\ Harvard-Smithsonian Center for Astrophysics, Cambridge, MA 02138 \\ Received 2006 January 22; accepted 2006 June 19
}

\begin{abstract}
We have obtained EUV spectra between 90 and $255 \AA$ of the comets C/2002 T7 (LINEAR), C/2001 Q4 (NEAT), and C/2004 Q2 (Machholz) near their perihelion passages in 2004 with the Cosmic Hot Interstellar Plasma Spectrometer (CHIPS). We obtained contemporaneous data on NEAT with the Chandra ACIS instrument, marking the first simultaneous EUV and X-ray spectral observations of a comet. The total CHIPS/EUV observing times were $337 \mathrm{ks}$ for NEAT, $234 \mathrm{ks}$ for LINEAR, and $483 \mathrm{ks}$ for Machholz, and for both CHIPS and Chandra we calculate we have captured all the comet flux in the instrument field of view. We set upper limits on solar wind charge-exchange emission lines of $\mathrm{O}, \mathrm{C}, \mathrm{N}, \mathrm{Ne}$, and Fe occurring in the spectral bandpass of CHIPS. The spectrum of NEAT obtained with Chandra can be reproduced by modeling emission lines of $\mathrm{C}, \mathrm{N}, \mathrm{O}, \mathrm{Mg}, \mathrm{Fe}, \mathrm{Si}$, $\mathrm{S}$, and $\mathrm{Ne}$ solar wind ions. The measured $\mathrm{X}$-ray emission-line intensities are consistent with our predictions from a solar wind charge-exchange model. The model predictions for the EUV emission-line intensities are determined from the intensity ratios of the cascading X-ray and EUV photons arising in the charge-exchange processes. They are compatible with the measured limits on the intensities of the EUV lines. For NEAT, we measured a total X-ray flux of $3.7 \times 10^{-12} \mathrm{ergs} \mathrm{cm}^{-2} \mathrm{~s}^{-1}$ and derive from model predictions a total EUV flux of $1.5 \times 10^{-12} \mathrm{ergs} \mathrm{cm}^{-2} \mathrm{~s}^{-1}$. The CHIPS observations occurred predominantly while the satellite was on the dayside of Earth. For much of the observing time, CHIPS performed observations at smaller solar angles than it was designed for, and EUV emission from the Sun scattered into the instrument limited the sensitivity of the EUV measurements.
\end{abstract}

Subject headings: comets: general — comets: individual (C/2002 T7 (LINEAR), C/2001 Q4 (NEAT), C/2004 Q2 (Machholz)) — ultraviolet: solar system

Online material: color figures

\section{INTRODUCTION}

Soft X-ray emission has been detected from at least 20 comets since 1996. After the consideration and rejection of several possible emission mechanisms, a consensus has been reached that the primary mechanism is charge-exchange collisions between highly charged solar wind minor ions and neutral atoms and molecules of the cometary atmospheres, as suggested by Cravens (1997). The observations have been reviewed by Cravens (2002), Krasnopolsky et al. (2004), and Lisse et al. (2004). The solar wind charge-exchange (SWCX) process also occurs in the heliosphere, where it contributes to the soft X-ray background (Cox 1988; Cravens 2000; Robertson \& Cravens 2003; Pepino et al. 2004; Lallement 2004) and may be responsible for some fraction of the short and- long-term enhancements seen at $250 \mathrm{eV}$ (Snowden et al. 1995, 2004). These recent, interesting observations indicate that it is important to understand both the microphysics of the charge-exchange process and the phenomenology of where, when, and how this process occurs within the solar system.

Theoretical models of the X-ray and EUV emission lines produced in comets by charge exchange with the solar wind ions have been constructed by Wegmann et al. (1998), Schwadron \& Cravens (2000), Kharchenko \& Dalgarno (2000, 2001), Kharchenko et al. (2003), Beiersdorfer et al. (2003), and Lisse et al. (2004).

Observations of diffuse SWCX emission other than from comets have been carried out using Chandra (Wargelin et al. 2004) and XMM-Newton (Snowden et al. 2004). Wargelin et al. (2004) observe the optically dark side of the Moon and attribute the 
X-rays they detect to charge-exchange collisions between solar wind ions and neutral hydrogen in the Earth's geocorona. Snowden et al. (2004) ascribe the emission they see as coming from the Earth's exosphere or the heliosphere. In both cases, the emission is characterized by strong variability and correlation with the solar wind particle flux. X-rays generated by SWCX have been detected from the atmospheres of Mars and Venus (Murawski \& Steinolfson 1996; Dennerl 2002; Holmstrom \& Kallio 2004; Fok et al. 2004; Gunell et al. 2004).

In summary, the highly ionized, heavy ions that stream out in the solar wind are a potential source of soft X-ray and EUV emission from within the solar system. Charge-exchange emission may occur, involving neutral atoms and molecules from comets entering the inner solar system, planetary exospheres, and neutral atoms in the interplanetary medium. A highly ionized solar wind ion may give rise to several photons, as the ion captures electrons sequentially in its flight from the Sun. A detailed ionization and density map of the heavy solar wind ions as a function of time and position is required for a complete description of the $\mathrm{X}$-ray and EUV emissions in the solar system. Measurements of SWCX emission from comets (and other measurements) can provide a useful probe of the density and ion composition of the solar wind.

Many SWCX lines are predicted to appear at EUV energies below the low-energy limit of Chandra and XMM-Newton (Kharchenko \& Dalgarno 2000, 2001; Kharchenko et al. 2003). Some of these EUV lines arise from ions not conclusively identified from emission at higher energies. These lines may be detected with CHIPS, a spectrometer sensitive in the EUV with a resolution of several hundred. The lines appear to be present in the Röntgensatellit (ROSAT) PSPC spectrum of C/1990 K1 (Levy) (Dennerl et al. 1997), which rises steeply at energies below 200 eV. Krasnopolsky \& Mumma (2001) observed comet C/1996 B2 (Hyakutake) at low resolution at EUV wavelengths and claimed detection of lines from $\mathrm{O}, \mathrm{C}, \mathrm{He}$, and $\mathrm{Ne}$. Kharchenko \& Dalgarno (2001) predict that, at lower energies, emission lines from the O vi doublet at 1032 and $1038 \AA$ should be the brightest far-ultraviolet (FUV) SWCX lines. A sensitive search for EUV and FUV emission lines in cometary SWCX was conducted with the Far-Ultraviolet Spectroscopic Explorer (FUSE) observatory (Weaver et al. 2002; Feldman 2005). Weaver et al. (2002) report a tentative detection of $1032 \AA$ emission lines from comet C/2000 WM1 (LINEAR). An alternative interpretation of this feature has been suggested by Feldman (2005) from FUSE observations of the brighter comet C/2001 Q4 (NEAT); they attribute this feature to $\mathrm{H}_{2}$ fluorescent emission lines pumped by radiation from $\mathrm{N}$ III and $\mathrm{O} v \mathrm{VI}$ ions in the solar corona. The nondetection of the brightest FUV O VI lines from SWCX may be because the relatively small field of view of FUSE allowed observation of only about 6000 $7000 \mathrm{~km}$ around the cometary nucleus. For the Q4 FUSE observations, the peak brightness of the charge-exchange EUV and X-ray emissions was shifted by more than this amount from the comet center, and hence the FUSE aperture may have missed it (Feldman 2005).

The favorable perigee passages of the bright comets $\mathrm{C} / 2001$ Q4 (NEAT) and C/2002 T7 (LINEAR) in 2004 May and Machholz in 2005 January provided an excellent opportunity to use the unique low-energy spectroscopic capabilities of CHIPS to study SWCX in comets at EUV wavelengths. Due to a combination of high solar activity and their close approaches, comets C/2001 Q4 (NEAT) and C/2002 T7 (LINEAR) are the brightest $\mathrm{X}$-ray targets available since C/Hyakutake in 1996 and 153P/2002 C1 (Ikeya-Zhang) in 2002. In this paper, we discuss the observational data from CHIPS and Chandra, describe the procedures by which we measure the line strengths, and discuss the implications for the cometary charge-exchange process.

\section{OBSERVATIONS AND DATA REDUCTION}

\subsection{Instrumental}

The CHIPS orbit is approximately circular at $600 \mathrm{~km}$ and an inclination of $94^{\circ}$, determined by the primary payload (ICESAT) of the rocket that launched the instrument. CHIPS is sensitive to the EUV band of 90-260 $\AA$, or 47-138 eV. With all six slits open, the spectrograph field of view is roughly rectangular at $5^{\circ} \times 25^{\circ}$ Any object smaller than a few degrees presents a "point" source to CHIPS and will be visible only through two slits at a time. The observations presented here were collected in wide-slit mode, with a peak resolution for diffuse light of $\sim 3.4 \AA$ FWHM, reaching $\sim 4 \AA$ at the edges of the bandpass.

Light from the comet enters the CHIPS spectrograph through two slits, each $1 \mathrm{~mm} \times 7 \mathrm{~cm}$. The light diverges onto an $8 \times 6 \mathrm{~cm}$ grazing-incidence cylindrical diffraction grating with a central groove density of 1800 lines $\mathrm{mm}^{-1}$. The groove density is varied across the grating surface to provide nearly stigmatic focusing in the dispersion direction near the center of the spectral band. Thus, the spectral resolution near the center of the band is limited by the slit width. At the ends of the band, the combined effects of optical aberration and defocusing contribute to the net spectral line width. For sources that are significantly smaller than the field of view of a slit, the resolution is independent of the angular size of the comet.

The grating rulings and baffling reject most non-EUV light, and thin-film filters placed close to the detector also attenuate out-of-band and scattered light. A diffuse emission line spans the full available detector height, typically passing through two different filter materials: aluminum, zirconium, or polyimide/boron. Measurements of an emission line that has the appropriate ratio of line flux from the two different filters spanned by the line encourage the belief that an actual spectral line has been detected and not some detector artifact.

The CHIPS in-orbit calibration is discussed in more detail in Hurwitz et al. (2005), and additional details of the science instrument and the CHIPSat satellite are presented in Hurwitz et al. (2003, 2005), Janicik et al. (2003), Marckwordt et al. (2003), Sirk et al. (2003), and Sholl et al. (2003).

\subsection{Comet Observations and Data Reduction}

A summary of the CHIPS observations of all three comets is presented in Table 1. We co-add individual comet observations in different subsets as described below. Individual pointings are of $\sim 20$ minute duration and, owing to the orbital geometry, nearly all observations took place while the satellite was on the dayside of Earth. The CHIPS mission has a nominal constraint of $72^{\circ}$ for the minimum boresight-to-Sun angle permitted during observations, both for spacecraft health reasons and to minimize the amount of scattered solar radiation entering the spectrometer. However, observing NEAT Q4 and LINEAR T7 near their time of maximum optical brightness required observations that violated this Sun-angle constraint and required the development and use of a new observing mode to accommodate the smaller solar angles, as well as observe a target moving in the sky. The two most important differences for this mode are a slightly reduced pointing accuracy and stability, since the main Sun sensor did not have the Sun in the field of view, and a possible increase in the amount of scattered solar EUV radiation present in the comet spectra.

Initial data reduction was carried out using the pipeline that is described in Hurwitz et al. (2005). We apply several filters to the 
TABLE 1

Summary of CHIPS Comet Observations

\begin{tabular}{|c|c|c|c|c|c|c|c|c|}
\hline $\begin{array}{l}\text { Comet } \\
\text { (1) }\end{array}$ & $\begin{array}{c}\text { Date Range } \\
\text { Observation } \\
\text { (CHIPS) } \\
(2)\end{array}$ & $\begin{array}{c}\text { Exposure } \\
\text { Time } \\
\text { Day } \\
\text { (s) } \\
\text { (3) }\end{array}$ & $\begin{array}{c}\text { Exposure } \\
\text { Time } \\
\text { Night } \\
\text { (s) } \\
(4)\end{array}$ & $\begin{array}{l}\text { Sun Angle } \\
\text { Range } \\
\text { (deg) } \\
(5)\end{array}$ & $\begin{array}{l}\text { Sun-Comet } \\
\text { Distance } \\
\text { Range } \\
\text { (AU) } \\
\text { (6) }\end{array}$ & $\begin{array}{l}\text { Earth-Comet } \\
\text { Distance } \\
\text { Range } \\
\text { (AU) } \\
(7)\end{array}$ & $\begin{array}{l}V \text { Magnitude } \\
\text { Range } \\
(8)\end{array}$ & $\begin{array}{c}0.25-1 \mathrm{keV} \text { Flux } \\
\left(10^{-12} \mathrm{ergs}\right. \\
\left.\mathrm{cm}^{-2} \mathrm{~s}^{-1}\right) \\
(9)\end{array}$ \\
\hline C/2001 Q4 (NEAT)............. & 2004 Apr 21 07:35-2004 May 21 07:39 & 331096 & 5832 & $78.1-69.0$ & $1.05-0.97$ & $0.58-0.56$ & $2.6-2.1$ & 3.7 \\
\hline C/2002 T7 (LINEAR) ......... & 2004 May 02 11:45-2004 May 31 03:41 & 219477 & 14415 & $40.0-72.8$ & $0.65-1.0$ & $0.76-0.55$ & $1.5-2.7$ & $\cdots$ \\
\hline C/2004 Q2 (Machholz) ...... & 2005 Jan 06 23:44-2005 Jan 28 22:58 & 441466 & 41158 & $130.3-106.7$ & $1.24-1.21$ & $0.35-0.47$ & $4.1-4.7$ & . \\
\hline
\end{tabular}

Note.-Col. 9 from Chandra observations of 2004 May 9.

raw data in producing the reduced spectra. Data are excluded when the overall detector count rate exceeds 80 events $\mathrm{s}^{-1}$, indicative of high charged-particle backgrounds. Data are also excluded when the detector high voltage is reduced (a normal, temporary response to transient high-background periods). A broad pulseheight filter is applied by the flight software. Approximately $40 \%$ of the telemetered events are then excluded by a low-pass pulseheight threshold in ground software, selectively reducing background because the low-amplitude events are overwhelmingly triggered by charged particles, rather than by photons.

Periodically pulsed "stimulation pins" outside the active field of view are used to register the event $(X, Y)$ coordinates to a common frame, thereby correcting for thermal drifts in the plate scale or axis zero points, and to determine the effective duration of the observation. Distortion is corrected using a preflight pinhole grid map. Small regions of known detector "hot spots" are excluded, the event coordinates are rotated so that the new $X$-axis closely corresponds to the spectral dispersion direction, and the spectra in each filter half are summed over the active detector height.

Once the final spectra (one each from the upper and lower detector halves) have been summed, these will typically contain counts from several background sources in addition to any signal from a comet. The primary backgrounds include particle events that are generally uniform across the detector, geocoronal $\mathrm{He}$ I $584 \AA$ photons that are scattered by the gratings and penetrate the Al filters, and for these comet observations, some EUV photons from the Sun that are multiply scattered from some structure on the satellite into the spectrometer. These backgrounds must be properly accounted for or subtracted before we can assess the amount of radiation from the comets that may be present.

We show in Figure 1 the summed spectra from all the individual NEAT Q4 observations, totaling 329,495 s of useful data. The upper curve is the polyboron/Al side of the detector, while the lower trace is the $\mathrm{Zr} / \mathrm{Al}$ side of the detector, lowered by 200 counts. The wavelength ranges of the various filters are indicated, as are the positions of the filter-supporting bars for each half. The continuum seen in the poly/B and $\mathrm{Zr}$ filters is primarily from particle events, while the Al filters show a significant additional smooth background from scattered geocoronal He I $584 \AA$ Å photons. The effective area of the spectrometer as a function of wavelength is shown in Sirk et al. (2003). We add together signals from one or both halves of the detector at a particular wavelength based on the filter transmission curves to maximize signal-to-noise ratio $(\mathrm{S} / \mathrm{N})$ at that wavelength or to exclude artifacts. The ranges included from each filter/half are indicated in Figure 1.

To determine the continuum against which to search for emission lines, we use a very deep flight charged-particle spectrum, scaled to match the local counts in the spectrum under analysis. The deep charged-particle spectrum generally contains many more events per wavelength interval than does the spectrum under analysis, and thus contributes relatively little to the shot noise in the line flux. Differences in the technique by which the scale factor is determined, for example, heavy smoothing versus fitting loworder polynomials, result in changes of $\sim 10 \%$ to the line-flux results. To determine the absolute "flatness" of the detector response, we analyzed a preflight photon flat field that was histogrammed like the flight spectra. Compared to a smooth polynomial, the flat-field spectrum showed a pixel-to-pixel variance only slightly greater than that expected for shot noise, where "pixel" refers to the width of the narrowest spectral features. The excess $1 \sigma$ variance above shot noise was $\sim 0.3 \%$ of the total signal. This factor is negligible in all the limits reported here, where shot noise from charged particles detected is the dominant uncertainty.

We measure the line flux of an individual line by adding all the counts that occur within one spectral resolution element of the line-rest wavelength, and subtracting the counts summed from the scaled background spectrum over the same limits.

The wavelength scale is based on preflight measurements, offset by a (constant) $\sim 1 \AA$ determined from the bright geocoronal He II $256.3 \AA$ feature, which is present in essentially all the observations. Temporal variations in the measured centroid of the He II feature show a dispersion of $\sim 0.2 \AA$. Periodic observations of the moon, which provide a weakly reflected solar spectrum that includes lines of Fe IX-Fe XII, confirm that the adopted wavelength scale provides a good fit near the center of the spectral band and support the view that the relative throughput of the filter panels is as measured preflight. The measured fluxes of both the He II feature and the lunar spectrum are in good agreement with preflight expectations, suggesting that the instrument throughput has not declined since laboratory calibration. The preflight laboratory throughput calibration tied to an NIST-calibrated diode, both at a component level and an end-to-end manner (which agree to within $\sim 10 \%$ ), provides the absolute flux calibration.

\subsection{Pointing Stability}

The CHIPS at attitude control system (ACS) is described in Janicik et al. (2003). The ACS uses inputs from several sensors, including coarse and medium Sun sensors, magnetic sensors, a lunar sensor, and reaction wheel rotation sensors to calculate the spacecraft attitude. During most of the observations reported here, CHIPS was unable to use its most accurate source of attitude information, the spacecraft's medium Sun sensor. Instead, the adopted observing procedure was to orient the spacecraft such that the Sun was in the Sun sensor for the fraction of each orbit when a comet was not being observed, then slew to the comet during the period when the comet was visible. When the Sun sensor is used, the typical pointing accuracy of the spacecraft is within $\sim 0.5$ of the requested target (Janicik et al. 2003) and 


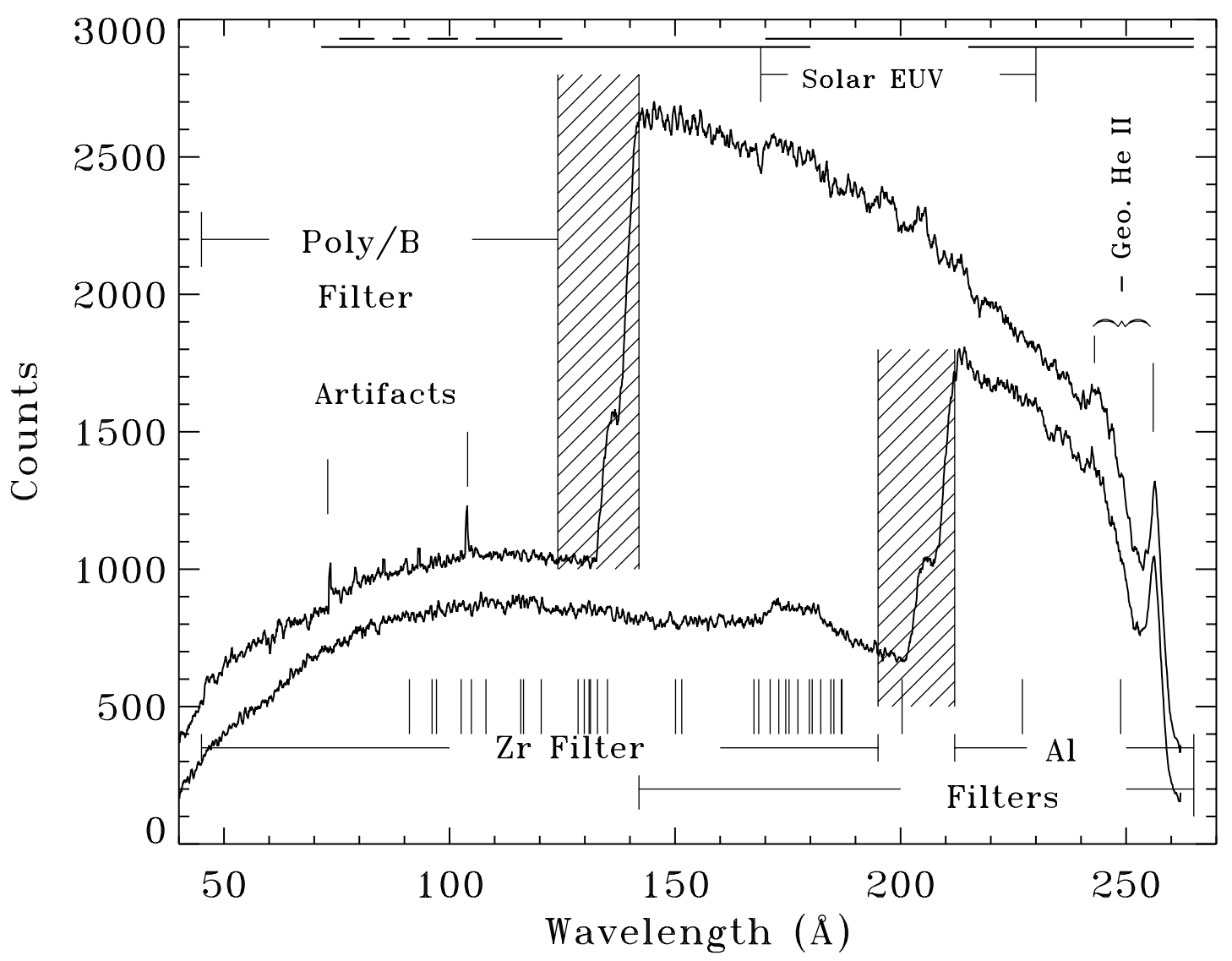

FIG. 1.-Complete CHIPS spectrum after pipeline processing using all data from C/2001 Q4 (NEAT) on the two detector halves, shown in counts. The lower spectrum has been displaced by 200 counts for clarity. The filter bandpasses and edges, location of known solar and geocoronal lines, and detector/light artifacts that show up in all CHIPS spectra are marked. Vertical marks show the location of charge-exchange lines predicted in this band from comets. Our flux detection limits for a particular line use one or both halves (indicated by lines at the top of the figure; Poly/ B/Al top, $\mathrm{Zr} / \mathrm{Al}$ bottom) to optimize the S/N at that wavelength.

centered to within $1 / 10$ of the spectrometer field of view. For relatively short periods, even with slews, such as a typical 25 minute comet observation, the spacecraft reaction wheels provide pointing orientation and knowledge, with negligible degradation. This is confirmed by the very small attitude corrections that followed reaquisition of the Sun by the medium Sun sensor.

In the CHIPS observations of the comets, we conclude that essentially all of the EUV-emitting region of the comet is contained within the CHIPS entrance apertures during the observations. The field of view of the two central slits of CHIPS is approximately $5 \mathrm{deg}^{2}$. Comet positions were generated every 10 minutes during the period they were observed by CHIPS using the Minor Plant and Comet Ephemeris Service, ${ }^{1}$ where the requested pointing corresponded to the midpoint in time of each observation. Parallax and the motion of the comet during an orbit's pointing are negligible compared with the CHIPS field of view. Our remapped image of Q4 on the Chandra ACIS-S detector shows the $\mathrm{X}$-ray-emitting region to be no larger than $10^{\prime} \times 10^{\prime}$. Given the attitude information and the size of the X-ray emitting region, we believe that the comets were positioned in the slit entrance aperture when the spacecraft reported that it was pointed at the requested comet position and that the emitting region was fully contained within the CHIPS field of view. Hence, for the observations reported here, we assume no dilution factor for comet EUV emission. The roll angle for observations was chosen such that the CHIPS entrance slits were perpendicular to the great circle that contained both the comet and the Sun.

\footnotetext{
${ }^{1}$ See http://cfa-www.harvard.edu/iau/MPEph/MPEph.html.
}

\subsection{Background/Noise Sources}

The majority of counts registered in the CHIPS detectors are from charged particles entering the instrument. Although some discrimination against particle events based on pulse height takes place as described above, the time-varying nature of this background makes it difficult to determine the presence of any continuum emission arising from photons. The net effect of the backgrounds and data-reduction techniques is that CHIPS is primarily sensitive to spectral lines, with a limiting sensitivity of a few line units or LU ( photons $\mathrm{cm}^{-2} \mathrm{~s}^{-1} \mathrm{sr}^{-1}$ ) for the observations described here.

CHIPS consistently detects at high-significance He II emission arising in the Earth's geocorona at $256.3 \AA$ and, to a lesser degree, at $243.0 \AA$. In addition, we observe radiation that appears to originate in the solar corona and is multiply scattered from surfaces on the spacecraft into the instrument. Although this background is very faint compared with the interstellar medium emission lines from Fe IX-Fe XII that CHIPS was designed to observe, we have studied it in depth, because it is a significant background to these measurements. The EUV line emission from the interstellar medium reported by Hurwitz et al. (2005) based on 1 yr of CHIPS observations is very faint and does not pose a significant background for the comet observations. A detailed study of the instrument-scattered background shows that the lines are never seen in co-added nighttime-only observations, and the spectrum appears very similar to the full-Moon spectrum. The strength of the lines does not correlate with the zenith angle, the lines show no ram-angle dependence, and they appear reproducibly in only a relatively small number of 


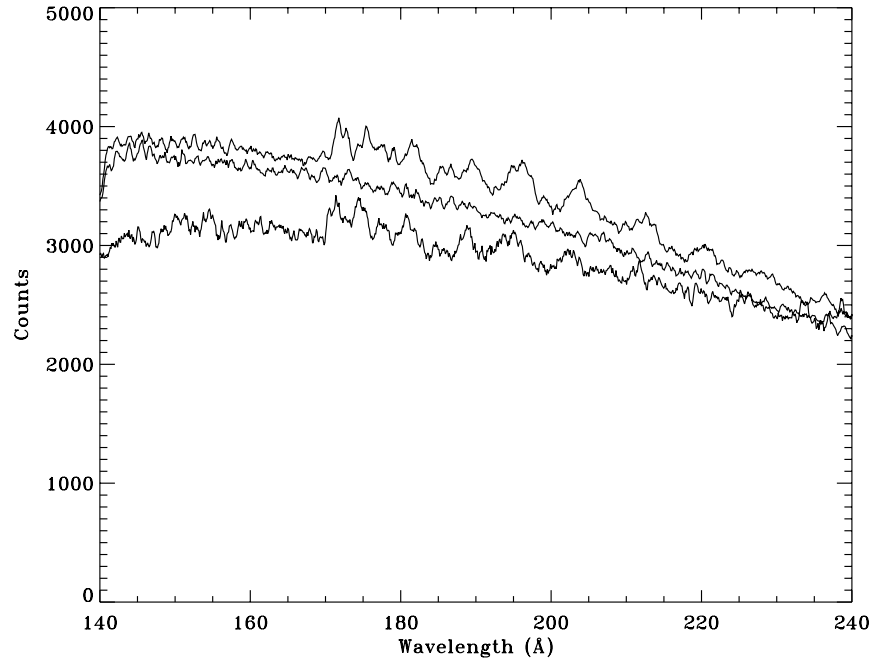

FIG. 2.-CHIPS dayside comet and lunar spectra through Al (polyimide/ boron detector half ) filter and standard pipeline processing. The spectra are not divided by the filter response. The upper trace shows a set of co-added CHIPS observations, selected when the dayside background lines are brightest. The middle trace shows a set of dayside spectra, chosen with low LFI, showing no solar contamination. The lowest trace shows the co-added full-Moon spectrum, taken for calibration purposes by CHIPS in narrow slit mode. The lines visible in the lunar spectrum arise from the solar corona being reflected by the Moon. The similarity in the spectral lines points to an origin in the solar corona for the dayside lines in the nonlunar spectra as well.

spacecraft orientations relative to the Sun. The lines appear more often, but not always, when CHIPS points closer to the Sun than during normal observations, but there is not a significant anticorrelation with the solar-target angle. The line strengths show no correlation with the Ultra Low Energy Isotope Spectrometer (ULEIS; Mason et al. 1998) measurements of Fe IX, Fe X, and Fe XI in the solar wind during the time of the comet observations, and the line strengths during comet observations do not correlate with measurements of solar disk irradiance in the 170-230 $\AA$ by the Solar EUV Experiment on TIMED (SEE; Woods et al. 1998). We conclude that the source of these background lines is faintly scattered radiation from the solar corona. Owing to its appearance preferentially at smaller solar angles, this background is therefore of more importance for the CHIPS comet observations than during normal sky survey observations, which are made at larger solar angles.

Figure 2 shows the co-added lunar spectrum observed by CHIPS in narrow-slit mode from several months in 2003 and the co-added spectrum from a number of observations, when the background lines were strongest. The similarity of the spectra is evident, although we would not expect identical spectra because the solar spectrum itself varies, and the scattered spectrum was taken in wide-slit mode, which is more sensitive to the scattered radiation and has lower spectral resolution. We can nonetheless use either the Moon spectrum or the co-added bright background times as a template to indicate the possible presence of solarscattered lines in the following way. We developed this indicator by first subtracting the continuum level from the scattered light spectrum after fitting it with a polynomial. We then similarly fit and subtract the continuum from another CHIPS observation. When the two continuum-subtracted spectra are multiplied together in the region of the lines, the template acts as a matched filter, sensitive to the solar lines. We call the exposure-timenormalized product of the two spectra integrated over the spectral region 170-230 A the "line-flux indicator," or LFI. We find

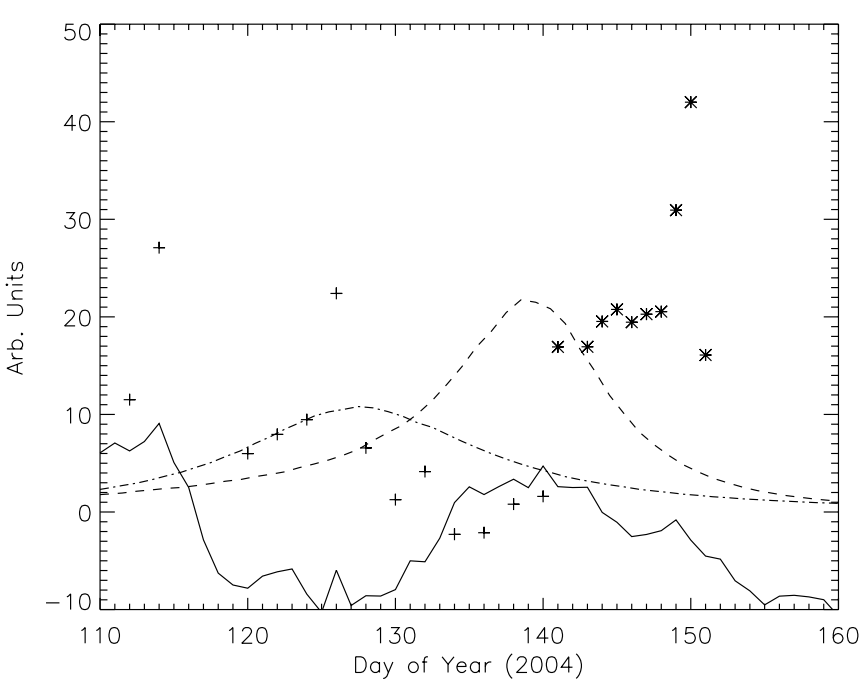

FIG. 3.- Several quantities related to the faint, variable background vs. day of year 2004 plotted in order to examine correlations with the background. The crosses indicate relative LFI (see text) strength by day for C/2001 Q4 (NEAT) observations, and the asterisks, for $\mathrm{C} / 2002 \mathrm{~T} 7$ (LINEAR) observations. The last six Q4 points are designated no-LFI points. The solid line is a scaled and offset measure of the SEE 170-230 ̊ flux. The relative observing geometry appears to be a larger determinant of LFI magnitude than the solar EUV flux as measured by SEE. We also show, to indicate the relative comet brightness where our observations were made, a geometry-only prediction (based on Horizons ephemeris) of comet flux, based on the distance from Earth and the Sun. The dot-dashed line is for $\mathrm{Q} 4$, and the dashed line is for $\mathrm{T} 7$.

the LFI to be a sensitive indicator of the presence of scattered solar light in co-added observations and can use it to reject data that contains this background where required. The third trace in Figure 2 is co-added daytime data selected for low LFI and shows essentially no solar contamination. However, in most cases we measure only upper limits to the spectral lines expected from charge-exchange reactions, so that the presence of a possible solar background is of less concern than it would be were CX lines detected. In addition, the $\mathrm{CX}$ lines we are trying to detect form an only partially overlapping set with the lines emerging from the solar corona.

We show in Figure 3 the relative LFI value for each day of the Q4 and T7 CHIPS observations. We also show the scaled magnitude of the solar 170-230 $\AA$ flux of the Sun as measured by SEE, which does not correlate with the LFI values. Finally, we also show a model of the visual brightness of the two comets, where the variation is based only on the inverse-squared distances from the Earth to the comet and the comet to the Sun. The latter curves indicate that we observed Q4 through its peak brightness, while the T7 observations were only possible as the brightness declined, since the comet was too close to the Sun in the sky to be observed prior to that time. Owing to the sensitivity level of the CHIPS data, it is not useful to subdivide the data into different time slices to investigate possible time variation of the emission.

\subsection{Chandra Observations of C/2001 Q4 (NEAT)}

Comet C/2001 Q4 (NEAT) was observed by Chandra on 2004 May 12 using director's discretionary time. The total approved observation of $10 \mathrm{ks}$ was divided into three pointings of approximately equal length. During each observation the telescope was held fixed, and the comet allowed to drift through the field of view. The comet was centered on the S3 chip, which has the best low-energy response. The three resultant standard level 2 event lists were simultaneously concatenated and reprojected 
TABLE 2

Charge-Exchange Line-Flux Limits

\begin{tabular}{|c|c|c|c|c|c|c|c|c|c|c|c|c|c|c|c|c|}
\hline \multirow[b]{2}{*}{ WAVELENGTH $(\AA)$} & \multirow[b]{2}{*}{ Ion } & \multicolumn{3}{|c|}{ C/2001 Q4 No-LFI } & \multicolumn{3}{|c|}{ C/2001 Q4 } & \multicolumn{3}{|c|}{$\mathrm{C} / 2002 \mathrm{~T} 7$} & \multicolumn{3}{|c|}{ C/2004 Q2 } & \multicolumn{3}{|c|}{ All Combined } \\
\hline & & Limit & $\sigma$ & Sign. & Limit & $\sigma$ & Sign. & Limit & $\sigma$ & Sign. & Limit & $\sigma$ & Sign. & Limit & $\sigma$ & Sign. \\
\hline $115.86 \ldots \ldots$ & $\mathrm{O}$ VI & -0.51 & 0.77 & 0.00 & 0.26 & 0.48 & 0.53 & 1.01 & 0.58 & 1.74 & 0.19 & 0.42 & 0.45 & 0.39 & 0.30 & 1.31 \\
\hline $120.33 \ldots$ & $\mathrm{O}$ VII & 0.20 & 0.76 & 0.27 & 0.14 & 0.47 & 0.29 & -0.83 & 0.56 & 0.00 & -0.62 & 0.41 & .00 & -0.42 & 0.29 & 0.00 \\
\hline $128.48 \ldots$ & $\mathrm{O}$ VII & 0.26 & 1.07 & 0.25 & 0.06 & 0.66 & 0.09 & 0.10 & 0.78 & 0.12 & 0.64 & 0.56 & .13 & 0.33 & 0.40 & 0.84 \\
\hline $129.91^{\mathrm{a}}$. & $\mathrm{O}$ VI & 2.22 & 1.16 & 1.92 & 0.52 & 0.71 & 0.73 & -0.34 & 0.83 & 0.00 & 0.56 & 0.60 & 0.93 & 0.35 & 0.43 & 0.82 \\
\hline $133.92 \ldots$ & $\mathrm{N}$ VII & -0.04 & 1.29 & 0.0 & 1.07 & 0.8 & 1.3 & 0.40 & 0. & 0. & -0.69 & 0.67 & 0.0 & 0.11 & 0.48 & 0.24 \\
\hline $135.02 \ldots \ldots$. & $\mathrm{C}$ VI & -0.27 & 1.31 & 0.00 & 0.27 & 0.81 & 0.34 & 0.34 & 0.95 & 0.36 & -0.08 & 0.68 & .00 & 0.12 & 0.48 & 0.26 \\
\hline $150.21 \ldots$ & $\mathrm{O}$ VI & -1.84 & 1.60 & 0.00 & -0.21 & 0.97 & 0.00 & -1.09 & 1.14 & 0.00 & 0.15 & 0.82 & 19 & -0.24 & 0.58 & 0.00 \\
\hline 173.29. & $\mathrm{O} \mathrm{VI}$ & 1.84 & 2.02 & 0.91 & 5.16 & 1.26 & 4.10 & 3.48 & 1.57 & 2.22 & 1.85 & 0.87 & 2.13 & 3.27 & 0.73 & 4.49 \\
\hline $182.28 \ldots$ & $\mathrm{C}$ VI & 0.64 & 2.56 & 0.25 & 2.76 & 1.63 & 1.69 & 6.13 & 2.11 & 2.91 & -0.69 & 1.02 & 0.00 & 1.90 & 0.95 & 2.00 \\
\hline $184.19 \ldots \ldots \ldots$ & $\mathrm{O}$ VI & -2.95 & 2.56 & 0.00 & -1.96 & 1.63 & 0.00 & -7.77 & 2.10 & 0.00 & 0.55 & 1.03 & 0.54 & -2.06 & 0.95 & 0.00 \\
\hline $186.74 \ldots \ldots \ldots \ldots \ldots$ & $\mathrm{C} v$ & 2.15 & 2.60 & 0.83 & -0.47 & 1.65 & 0.00 & -0.47 & 2.14 & 0.00 & 1.59 & 1.04 & 1.53 & 0.48 & 0.97 & 0.50 \\
\hline
\end{tabular}

Note.-Limit and local $\sigma$ values in LU. Sign. $=$ limit $/ \sigma$. To find a limit in photons $\mathrm{cm}^{-2} \mathrm{~s}^{-1}$, multiply the value in LU by 0.0106 .

${ }^{a}$ Indicated lines and those with $\lambda>170 \AA$ are subject to contamination from solar Fe vIII-Fe XII emission.

into a fixed "comet-centered" frame of reference using the CIAO ${ }^{2}$ tool sso_freeze. The final reconstructed image provides good spatial $\left(\sim 0.5\right.$ pixel $\left.^{-1}\right)$ and spectral information $(\sigma \sim 50 \mathrm{eV})$.

A source spectrum was extracted from a circular aperture with a radius of 700 pixels (5.8) centered on the peak of the comet emission, and the background spectra were extracted from the area outside the source aperture. We examined how varying the aperture radius changed the comet and background spectrum. The aperture radius we chose included essentially all of the emission from the comet; outside of this radius, we expect that heliospheric SWCX will be a uniform background on this scale. The background spectrum normalized by area was subtracted from the in-aperture spectrum to produce the Q4 comet spectrum. Work by Lisse et al. (1996) and Wegmann \& Dennerl. (2004) shows that the CX X-ray emission from a comet as active as Q4 may be offset from the nucleus by $30,000-40,000 \mathrm{~km}$. At the distance of 0.6 AU, where Q4 was observed with Chandra, this separation translates to an angle of $\sim 1^{\prime}$. Owing to the diffuse nature of the emission and the positional uncertainty induced by the photon remapping, it is not clear whether we can discern a potential offset of this magnitude from the center of the nucleus, but we have verified that the spectrum resulting from our chosen aperture radius is insensitive to a potential offset of this size. A detailed discussion of the Q4 X-ray emission morphology will be covered in C. M. Lisse et al. (2007, in preparation). ACIS response matrices for modeling the instrument-effective area and energy-dependent sensitivity were created with the standard CIAO tools, and the resulting spectra were fit using XSPEC.

\section{RESULTS AND DISCUSSION}

Figure 1 shows the C/2001 Q4 (NEAT) spectrum from 50 to $270 \AA$ measured with CHIPS. For each potentially strong chargeexchange spectral line indicated in the figure, we measure the brightness using the spectra from one or both halves of the CHIPS detector. We have run our line-flux measuring algorithm for each comet, and the results of these measurements are shown in Table 2.

\footnotetext{
${ }^{2}$ Chandra Interactive Analysis of Observations.
}

We include two measurements using the Q4 data: (1) the "no-LFI" set, referring to the period 2004 May 9-19, corresponding to a time when the LFI measurement indicates minimal contamination from scattered solar EUV radiation and (2) the line limits when using all the Q4 CHIPS data. We also present the limits from a spectrum consisting of the co-added spectra from all three comets, which contains the lowest relative shot-noise background. We measure positive flux at the wavelengths of a number of potential charge-exchange lines, albeit at low significance.

Fe lines at wavelengths 170-220 $\AA$ are detected in several of the spectra at significances ranging to $5 \sigma$. Since it is likely that much or all of this emission is solar rather than cometary, we restrict our consideration to possible charge-exchange lines shortward of $170 \AA$, although line measurements that may have a solar component provide upper limits to the cometary emissions. We estimate that the lines for which we report limits in the table contribute about $90 \%$ of the flux from charge exchange, if we ignore the iron lines.

The least contaminated spectrum is that obtained from the NEAT Q4 data selected for low LFI, and the most significant line is the $\mathrm{O}$ VI line at $129.91 \AA$, at almost $2 \sigma$, arising from the $1 s 4 d^{3} D-1 s 2 p^{3} P$ transition.

In Figure 4, we show the results, binned to the CHIPS instrumental resolution, of the line-flux measuring algorithm run on a $1 \AA$ wavelength grid on the combined data set. When using this technique, a positive actual line flux suppresses the neighboring continuum. Hence, positive flux should be read from the graph axis, not the adjacent continuum. Tentative line identifications of features associated with positive flux measurements are indicated. The feature at 91.1 $\AA$ coincides with the transition $1 s 4 p^{3} P-1 s 2 s^{3} S$ of $\mathrm{O}$ VII, and the feature at $128.5 \AA$ to the $1 s 3 d^{3} D-1 s 2 p^{3} P$ transition. O vi has lines at $116.4,129.9,132.5$, and $150.1 \AA$, corresponding respectively to the $4 p-2 s, 4 d-2 p, 4 s-2 p$, and $3 p-2 s$ transitions.

A search for line detections can also be conducted using theoretical predictions for the wavelength and flux ratio of several lines from the same ion. We employed the nine brightest lines in the CHIPS band of the $\mathrm{O}$ vI ion given by the model described in 


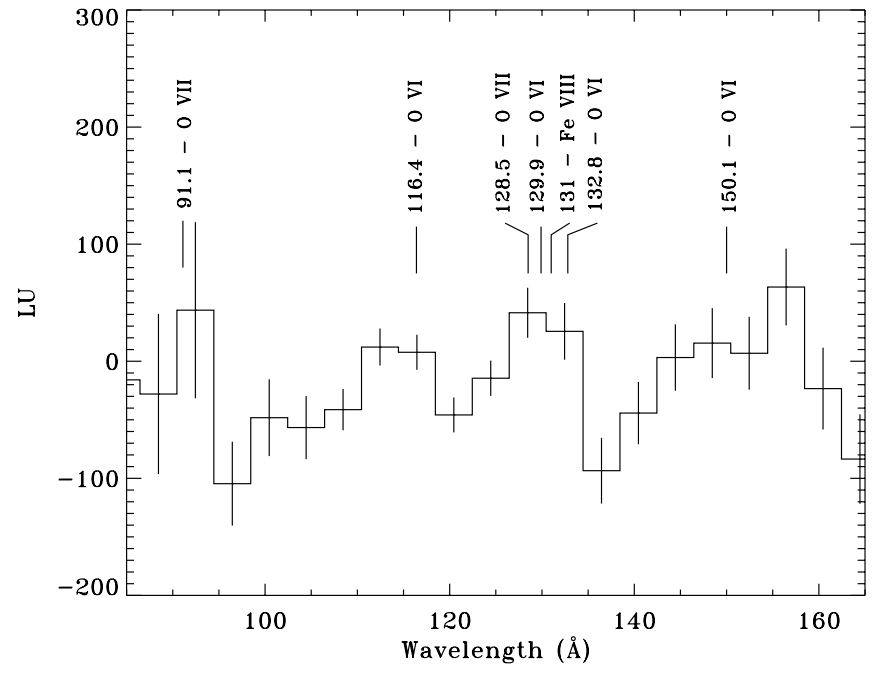

FIG. 4.-All three comet spectra summed, then continuum subtracted. This procedure can improve the visibility of strong emission lines, but is subject to uncertainties in the continuum level placement. Spectra and errors shown at $4 \AA$ resolution, close to the actual instrument resolution, and the error bars are $1 \sigma$. Some tentative spectral line identifications are indicated. Longward of $170 \AA$, solar Fe lines dominate the spectrum.

the following section. The line profiles were taken to be Gaussian, with widths equal to the wavelength-dependent resolution of CHIPS. The free parameters in the fit were a quintic polynomial used to find the background level and an overall amplitude for the $\mathrm{O}$ vi lines, with the individual line ratios fixed. The results when this technique was applied to the NEAT Q4 no-LFI spectrum are that the line amplitudes are consistent with zero. We carried out the same procedure for the co-added comet $\mathrm{T} 7$ spectra, and again the results were consistent with zero $\mathrm{O}$ vi emission.

\section{MODELING THE SOLAR WIND CHARGE-EXCHANGE EMISSION}

The NEAT Q4 count spectrum obtained from Chandra is shown in Figure 5. It contains emission lines seen in other comets (Lisse et al. 2001; Krasnopolsky et al. 2002). We first use XSPEC to fit the spectrum with eight emission lines whose wavelengths were allowed to vary. The best fit was achieved with eight lines at energies in eV of $245,320,403,496,570,655,830$, and 952, with corresponding photon fluxes in units of $10^{-4}$ photons $\mathrm{cm}^{-2} \mathrm{~s}^{-1}$ of, respectively, 3.68, 26.5, 6.57, 2.59, 5.93, 1.05, 0.29, and 0.11. The resulting $\chi^{2}$ per degree of freedom was 0.91 . The strong lines at 320,403 , and $570 \mathrm{eV}$ correspond to transitions of C v, N vi, and $\mathrm{O}$ vII. The lines near $245 \mathrm{eV}$ may be from $\mathrm{Si}$ IX, $\mathrm{Mg}$ IX, or $\mathrm{Mg} \mathrm{x}$, and a feature at $952 \mathrm{eV}$, found also in the spectra of comets McNaught-Hartley and LINEAR 4 (Krasnopolsky et al. 2002, 2004), may be from Ne IX. There is an indication of a line near $835 \mathrm{eV}$, but we hesitate to attribute it to the $5 p-1 s$ resonance line of $\mathrm{O}$ VIII, because we do not find the corresponding $2 p-1 s$ line at $653 \mathrm{eV}$, which we predict should be more intense by a factor of 6 . Because of the low resolution of the instrument, fits with a similar $\chi^{2}$ figure of merit are also obtained by a combination of emission lines and thermal bremsstrahlung at a temperature of about $0.23 \mathrm{keV}$, as in Kharchenko \& Dalgarno (2000).

We have extended a model developed by Kharchenko \& Dalgarno (2000, 2001), Kharchenko et al. (2003), and Pepino et al. (2004) to compute emission spectra over the full EUV and $\mathrm{X}$-ray spectral range. In the model, we use electron-capture cross sections for positive ions colliding with neutral $\mathrm{H}_{2} \mathrm{O}$ molecules

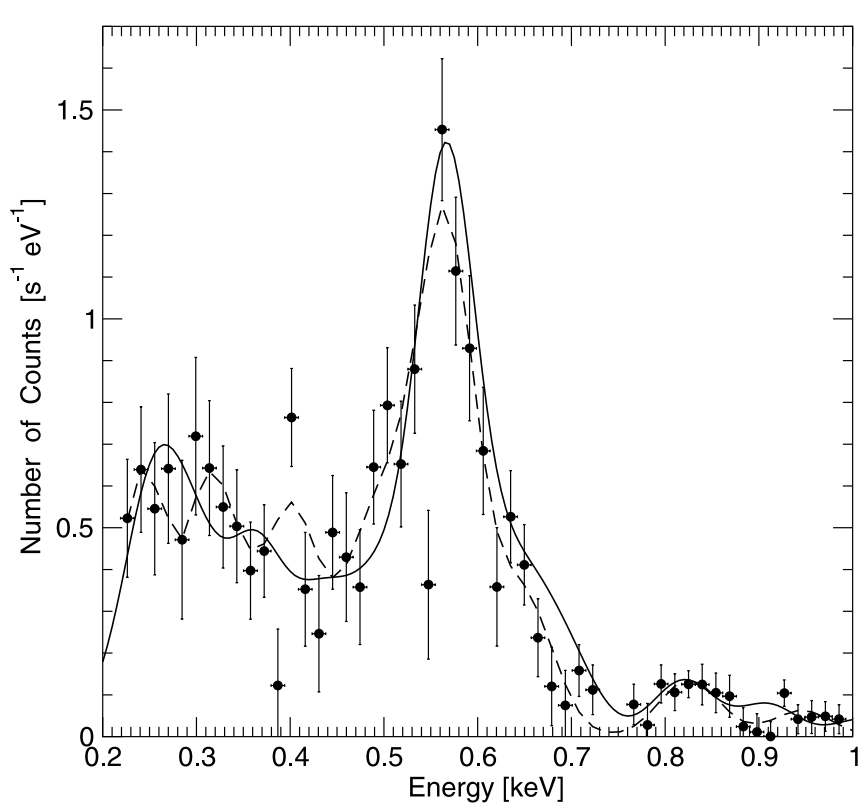

FIG. 5.-Two models of X-ray emission lines are compared with the Chandra spectrum for C/2001 Q4 (NEAT). The filled circles are the number counts from Chandra data. The dashed curve is the Chandra data, interpreted using an XSPEC fit with 8 lines between 245 and $952 \mathrm{eV}$, folded through the instrument response. The solid line shows the SWCX model predictions at a resolution of $72 \mathrm{eV}$. While both models yield an acceptable fit from a $\chi^{2}$ standpoint, only the SWCX model is based on substantially all of the underlying physics of the emission process. [See the electronic edition of the Journal for a color version of this figure.]

that reproduce the experimental laboratory X-ray spectra when available and theoretical estimates when they are not (Rigazio et al. 2002). When applied to the measured spectra, the method takes into account empirically determined processes, such as multiple capture followed by autoionization. The capture cross sections determine the entry rates into the individual excited states of the ion created in the charge exchange. The excited states decay in a radiative cascade which produces photons at X-ray, EUV, and UV wavelengths. The relative intensities of the emission lines occurring in the cascade from a given initial level depend only on the branching ratios of the transition probabilities. Thus, the EUV spectrum is determined by the X-ray spectrum, and the comet spectra depend on and provide a measure of the solar wind ion composition.

\subsection{X-Ray Emission Lines}

We calculated the X-ray emission-line intensities for the ion composition listed in Table 3. It is based on the ion composition of Schwadron \& Cravens (2000) for a fast solar wind, supplemented by the presence of a fraction of $0.001 \%$ of highly charged Fe ions, Fe xv-Fe xx, that appear to be necessary to reproduce the observed spectrum between 0.8 and $0.9 \mathrm{keV}$. An enhanced flux of highly charged iron ions may be a consequence of fast coronal mass ejection events (Lepri \& Zurbuchen 2004; Zurbuchen et al. 2004).

A comparison of the observations in counts per second of NEAT Q4 and the XSPEC and SWCX models at photon energies between 0.3 and $1.0 \mathrm{keV}$ is presented in Figure 5. The Chandra data with the error bars indicating $1 \sigma$ uncertainties are shown as filled circles. The dashed line is the XSPEC model fit, with eight emission lines folded through the instrument response. The solid line is the SWCX prediction at a spectral resolution of $75 \mathrm{eV}$. In Table 4, we list the energies and wavelengths and the predicted 
TABLE 3

SOLAR Wind Ion Composition

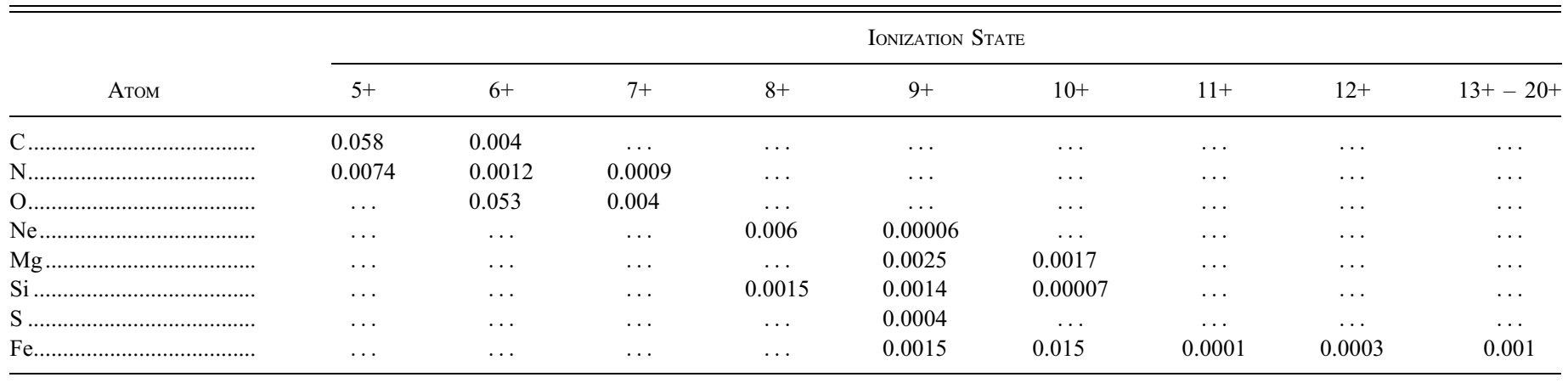

NoтES.-Fractional abundance by number density for each ion used for X-ray and EUV model spectrum. Blank columns indicate that zero abundance was used for the model spectrum.

relative intensities of the principal X-ray and EUV emission lines of the SWCX model. The agreement of the measurements of the X-ray lines with the models is good, but because of the low spectral resolution, we cannot exclude a significant contribution from bremsstrahlung from XSPEC modeling of the X-ray lines alone.

\subsection{EUV Emission Lines}

The photon fluxes measured for NEAT Q4 with CHIPS at energies below $150 \mathrm{eV}$ and with Chandra at energies between $250 \mathrm{eV}$ and $1.0 \mathrm{keV}$ are compared in Figure 6 with the predictions of the SWCX model. A scaling factor has been applied to bring the predicted total flux of the X-ray emission with energies greater than $250 \mathrm{eV}$ into agreement with the measured value of $3.7 \times$ $10^{-12} \mathrm{ergs} \mathrm{cm}^{-2} \mathrm{~s}^{-1}$. Table 4 can then be used to yield the absolute intensities shown in Figure 6. The corresponding total flux of the EUV lines between 47 and $140 \mathrm{eV}$ is $1.5 \times 10^{-12} \mathrm{ergs} \mathrm{cm}^{-2} \mathrm{~s}^{-1}$, distributed among 181 lines of $\mathrm{C}, \mathrm{N}, \mathrm{O}, \mathrm{Ne}$, and Fe. The theo-

TABLE 4

Relative Intensities of Emission Lines

\begin{tabular}{|c|c|c|c|}
\hline $\begin{array}{l}\text { Energy } \\
(\mathrm{eV})\end{array}$ & Relative Intensity & Ion & Transition \\
\hline $47.65 \ldots \ldots$ & 0.19 & $\mathrm{Cv}$ & $1 s 3 s{ }^{3} S-1 s 2 p{ }^{3} P$ \\
\hline 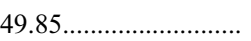 & 0.34 & $\mathrm{Cv}$ & $1 s 3 d^{3} D-1 s 2 p^{3} P$ \\
\hline 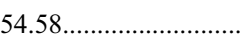 & 0.30 & $\mathrm{Cv}$ & $1 s 3 p^{3} P-1 s 2 s^{3} S$ \\
\hline $71.56 \ldots \ldots \ldots \ldots \ldots$ & 0.50 & $\mathrm{O} \mathrm{VI}$ & $3 d-2 p$ \\
\hline $82.56 \ldots \ldots \ldots \ldots$ & 0.20 & $\mathrm{O}$ VI & $3 p-2 s$ \\
\hline $93.70 \ldots \ldots \ldots \ldots \ldots . .$. & 0.31 & $\mathrm{O}$ VI & $4 s-2 p$ \\
\hline $95.43 \ldots \ldots \ldots \ldots \ldots \ldots \ldots$ & 0.19 & $\mathrm{O}$ VI & $4 d-2 p$ \\
\hline 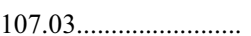 & 0.41 & $\mathrm{O}_{\mathrm{VI}}$ & $4 p-2 s$ \\
\hline 298.96 & $1^{\mathrm{a}}$ & $\mathrm{C} \mathrm{v}$ & $1 s 2 s^{3} S-1 s^{2}{ }^{1} S$ \\
\hline 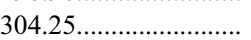 & 0.07 & $\mathrm{C} \mathrm{v}$ & $1 s 2 p^{3} P-1 s^{2}{ }^{1} S$ \\
\hline 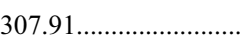 & 0.16 & $\mathrm{C} \mathrm{v}$ & $1 s 2 p{ }^{1} P-1 s^{2}{ }^{1} S$ \\
\hline 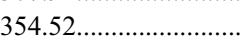 & 0.07 & $\mathrm{C} \mathrm{v}$ & $1 s 3 p^{1} P-1 s^{2}{ }^{1} S$ \\
\hline $367.35 \ldots \ldots \ldots \ldots \ldots \ldots \ldots$ & 0.11 & $\mathrm{C}$ VI & $2 p-1 s$ \\
\hline 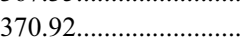 & 0.03 & $\mathrm{C} \mathrm{v}$ & $1 s 4 p{ }^{1} P-1 s^{2}{ }^{1} S$ \\
\hline $419.80 \ldots \ldots \ldots \ldots \ldots \ldots \ldots$ & 0.03 & $\mathrm{~N}$ VI & $1 s 2 s^{3} S-1 s^{2}{ }^{1} S$ \\
\hline 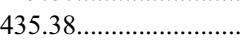 & 0.03 & $\mathrm{C}_{\mathrm{VI}}$ & $3 p-1 s$ \\
\hline 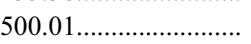 & 0.03 & $\mathrm{~N}$ VII & $2 p-1 s$ \\
\hline $560.86 \ldots \ldots \ldots \ldots \ldots \ldots \ldots$ & 0.14 & $\mathrm{O}$ VII & $1 s 2 s^{3} S-1 s^{2}{ }^{1} S$ \\
\hline
\end{tabular}

Notes.-The transitions with the highest emission by photon number in the model spectrum. The electronic configurations shown in the table for $\mathrm{Li}-$ and $\mathrm{H}$-like ions represent transitions for all states of electronic doublets.

${ }^{\mathrm{a}}$ The intensity of the $\mathrm{C} v$ line corresponds to a photon flux of $3.3 \times$ $10^{-3}$ photons $\mathrm{cm}^{-2} \mathrm{~s}^{-1}$. retical EUV intensities fall well below the measurements except near $95 \mathrm{eV}$, a region in which several lines of $\mathrm{O}$ VI and $\mathrm{O}$ VII appear. The most interesting CHIPS measurement of the SWCX lines is the $2 \sigma$ detection in the NEAT Q4 no-LFI spectrum of the line of $\mathrm{O}$ vi at $95.5 \mathrm{eV}$, or $130 \AA$. Discrepancies at lower energies are affected by scattered radiation from the Sun. We conclude that the SWCX model EUV predictions are consistent with the CHIPS data, but the instrument lacks the sensitivity needed to systematically test the theoretical predictions. In that CHIPS was not

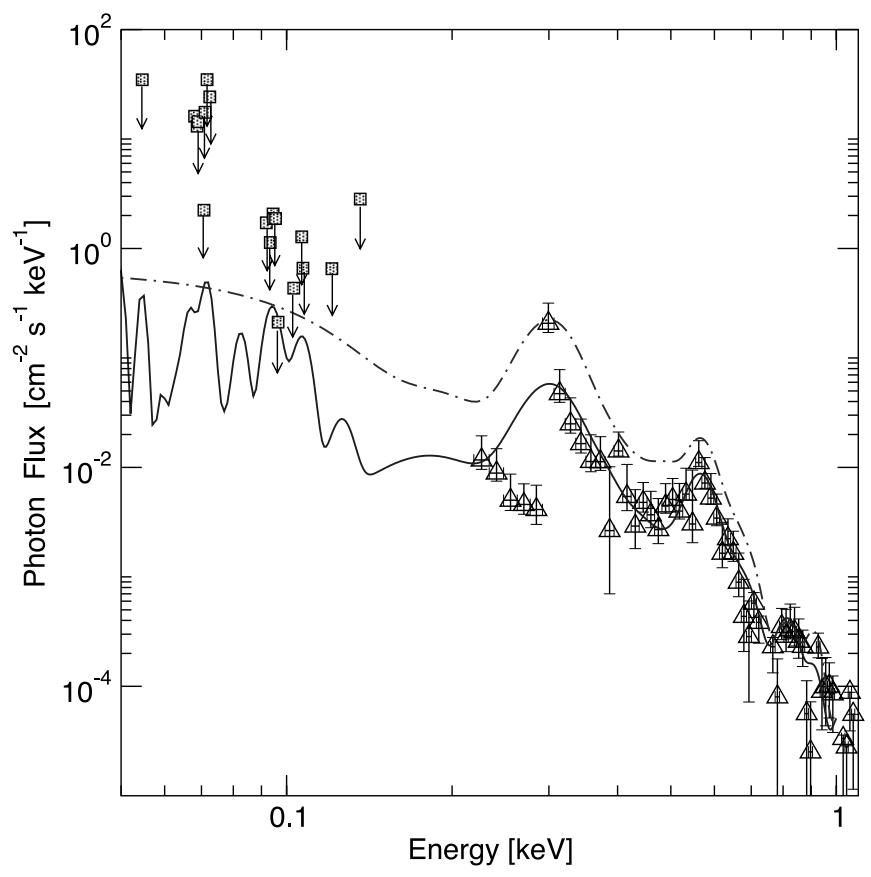

FIG. 6.- Intensity of the EUV and X-ray emission induced by the solar wind ions interacting with the atmosphere of Comet C/2001 Q4 (NEAT). The solid curve shows the prediction of the CX model for the photon flux in the energy interval of $0.047-1.1 \mathrm{keV}$. Absolute intensities of the theoretical CX spectra are normalized to the total number of X-ray photons observed with the Chandra telescope in the energy interval $0.25-1 \mathrm{keV}$. The triangles with error bars represent Chandra observations, and the squares show the result of observations with CHIPS. Theoretical X-ray spectra are computed for a FWHM of $70 \mathrm{eV}$, which corresponds to the Chandra observations. The resolution of the EUV spectral lines between 47 and $138 \mathrm{eV}$ has been taken to be equal to the CHIPS resolution of $4 \AA$. The dot-dashed line is the model fit to the unsubtracted comet $\mathrm{X}$-ray spectrum, including both comet and heliospheric charge-exchange components; it shows a predicted absolute upper limit to the SWCX emission. The EUV part of this curve is displayed with the same resolution as the X-ray portion. [See the electronic edition of the Journal for a color version of this figure.] 


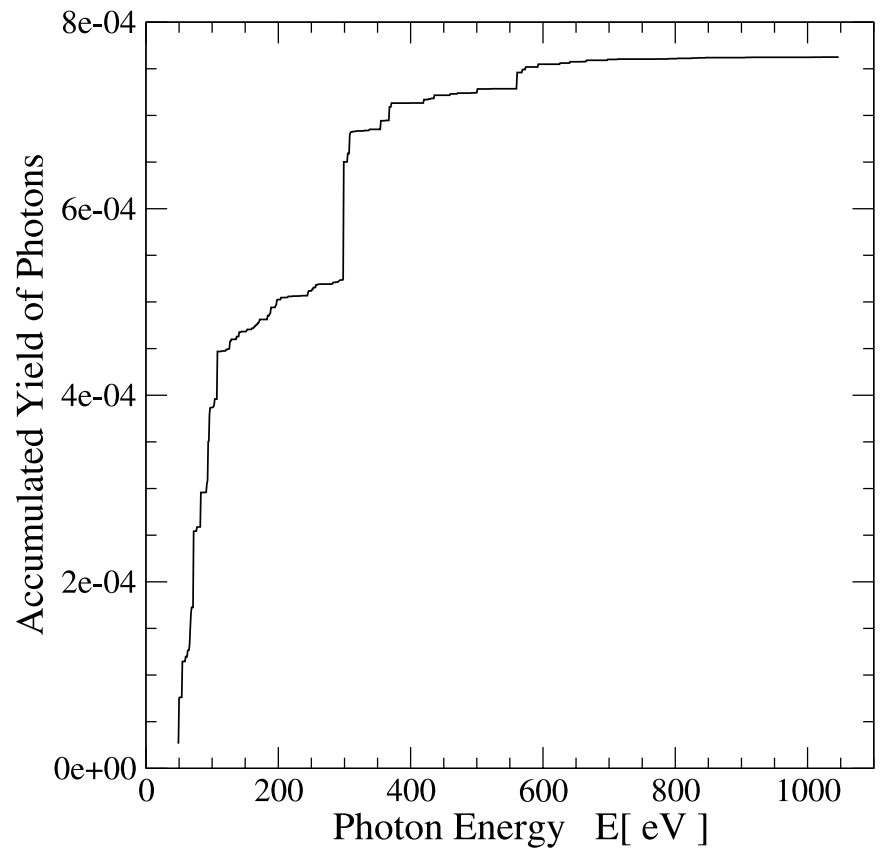

FIG. 7.- Predicted accumulated yield of EUV and X-ray photons for a single charge-exchange collision of the heavy solar wind ions, calculated from the SWCX model. The figure shows the average number of photons, induced with energies between $47 \mathrm{eV}$ and $E[\mathrm{eV}]$ in a single $C X$ collision of the solar wind ions with molecules of a cometary gas. The averaging procedure takes into account the relative abundances of the solar wind protons, $\alpha$-particles and heavy ions, and individual $\mathrm{CX}$ cross sections.

designed for measurements of comets or point sources, the limited agreement supports the SWCX model and points out some additional utility of the CHIPS measurements. The model also predicts that the 10 brightest EUV lines in the CHIPS band contribute $65 \%$ of all the EUV photons, and $88 \%$ of the EUV photon flux is contained in the brightest 26 lines.
The accumulated yield is useful in providing an estimate of the photon energy distribution from comets. It is defined as the total number of photons produced by charge exchange in collisions of the solar wind with comets that have energies lying between a minimum energy, equal to $47 \mathrm{eV}$ for CHIPS, and a maximum energy $E$. The accumulated yield is dimensionless. Multiplied by the product of the solar wind flux and ion density, it yields the volume emissivity. Figure 7 shows the calculated accumulated yield for NEAT Q4 as a function of photon energy $E$ for a minimum energy of $47 \mathrm{eV}$. Multiplying it by a factor of 26 yields the accumulated photon flux in units of $\mathrm{cm}^{-2} \mathrm{~s}^{-1}$. The small values reflect the low fractional abundances of the heavy solar wind ions.

\section{CONCLUSIONS}

We have measured EUV emission from three comets and provided the first-ever simultaneous EUV and X-ray measurement and modeling of cometary SWCX emission. The X-ray data for comet Q4 show clear emission lines that are satisfactorily interpreted as solar wind charge-exchange lines from ions expected in the solar wind. We have used the same model to identify and predict the strengths of EUV lines. The measured EUV line strengths are consistent with the theoretical model that reproduces the X-ray line intensities. The CHIPS data and model results show that the EUV lines from all three comets are quite faint. Nonetheless, the high spectral resolution measurements of the EUV lines have now set useful limits on many emission lines in this band that may serve as a guide for future EUV observations of comets.

The CHIPS team gratefully acknowledges support by NASA grant NAG 5-5213. A. D. and V. K. have been supported in this project by NASA grants NAG 5-1331 and NNG04GD57G. We thank the SEE PI team and NASA for making the SEE data available.
Beiersdorfer, P., et al. 2003, Science, 300, 1558

Cox, D. P. 1998, in IAU Colloq. 166, The Local Bubble and Beyond, ed.

D. Breitschwerdt, M. J. Freyberg, \& J. Trumper (New York: Springer), 121

Cravens, T. E. 1997, Geophys. Res. Lett., 24, 105

-. 2000, ApJ, 532, L153

2002, Science, 296, 1042

Dennerl, K. 2002, A\&A, 394, 1119

Dennerl, K., Englhauser, J., \& Trumper, J. 1997, Science, 277, 1625

Feldman, P. 2005, Phys. Scr. T, 119, 7

Fok, M.-C., Moore, T. E., Collier, M. R., \& Tanaka, T. 2004, J. Geophys. Res., 109, A01206

Gunell, H., Holmstrom, M., Kallio, E., Janhunen, P., \& Dennerl, K. 2004, Geophys. Res. Lett., 31, L22801

Holmstrom, M., \& Kallio, E. 2004, Adv. Space Res., 33, 187

Hurwitz, M. 2003, Proc. SPIE, 5164, 24

Hurwitz, M., Sasseen, T. P., \& Sirk, M. M. 2005, ApJ, 623, 911

Janicik, J., \& Wolff, J. 2003, Proc. SPIE, 5164, 31

Kharchenko, V., \& Dalgarno, A. 2000, J. Geophys. Res., 105, 18351 .2001, ApJ, 554, L99

Kharchenko, V., Rigazzio, M., Dalgarno, A., \& Krasnopolsky, V. 2003, ApJ, $585, \mathrm{~L} 73$

Krasnopolsky, V., 2004, Icarus, 167, 417

Krasnopolsky, V., Greenwood, J. B., \& Stancil, P. C. 2004, Space Sci. Rev., 113,271

Krasnopolsky, V., \& Mumma, M. J. 2001, ApJ, 549, 629

Krasnopolsky, V., et al. 2002, Icarus, 160, 437

Lallement, R. 2004, A\&A, 418, 143

\section{REFERENCES}

Lepri, S. T., \& Zurbuchen, T. H. 2004, J. Geophys. Res., 109, A01112

Lisse, C. M., Christian, D. J., Dennerl, K., Meech, K. J., Petre, R., Weaver, H. A., \& Wolk, S. J. 2001, Science, 292, 1343

Lisse, C. M., Cravens, T. E., \& Dennerl, K. 2004, in Comets II, ed. M. C. Festou, H. U. Keller, \& H. A. Weaver (Tucson: Univ. of Arizona Press), 631 Lisse, C. M., et al. 1996, Science, 274, 205

Marckwordt, M., et al. 2003, Proc. SPIE, 5164, 43

Mason, G. M., et al. 1998, Space Sci. Rev., 86, 409

Murawski, K., \& Steinolfson, R. S. 1996, J. Geophys. Res., 101, 2547

Pepino, R., Kharchenko, V., Dalgarno, A., \& Lallement, R. 2004, ApJ, 617, 1347

Rigazio, M., Kharchenko, V., \& Dalgarno, A. 2002, Phys. Rev. A, 66, 064701

Robertson, I. P., \& Cravens, T. E. 2003, Geophys. Res. Lett., 30, 22

Schwadron, N. A., \& Cravens, T. E. 2000, ApJ, 544, 558

Sholl, M., et al. 2003, Proc. SPIE, 5164, 63

Sirk, M. M., et al. 2003, Proc. SPIE, 5164, 54

Snowden, S. L., Collier, M. R., \& Kuntz, K. D. 2004, ApJ, 610, 1182

Snowden, S. L., et al. 1995, ApJ, 454, 643

Wargelin, B. J., Markevitch, M., Juda, M., Kharchenko, V., Edgar, R., \& Dalgarno, A. 2004, ApJ, 607, 596

Weaver, H. A., Feldman, P. D., Combi, M. R., Krasnopolsky, V., Lisse, C. M., \& Shemansky, D. E. 2002, ApJ, 576, L95

Wegmann, R., \& Dennerl, K. 2004, A\&A, 428, 647

Wegmann, R., Schmidt, H. U., Lisse, C. M., Dennerl, K., \& Englhauser, J. 1998, Planet. Space Sci., 46, 603

Woods, T. N., et al. 1998, Proc. SPIE, 3442, 180

Zurbuchen, T. H., et al. 2004, Geophys. Res. Lett., 31, L11805 